\begin{tabular}{ll} 
C O L L O Q U I U M & M A T H E M A T I C U M \\
\hline VOL. LXVIII & 1995 \\
\hline
\end{tabular}

\title{
THE DUGUNDJI EXTENSION THEOREM \\ AND EXTENSION DEGREE
}

BY

\section{KATSUYA EDA (TOKYO)}

1. Introduction. Generalizing the Tietze extension theorem, J. Dugundji [7] proved that any locally convex topological linear space $L$ is an absolute extensor for metrizable spaces, i.e. for any metrizable space $X$ and its closed subset $A$, every continuous map from $A$ to $L$ extends over $X$. To clarify the use of local convexity in the proof, we introduce a new notion of extension degree $\mathrm{D}(A, X)$ for a closed set $A$ in $X$. According to the definition below, $\mathrm{D}(A, X)=0$ if and only if $A$ is a retract of $X$. As we shall see in Theorem 1.2, this notion is strongly related to the essentiality of local convexity in the Dugundji theorem.

Now, we state the definition of the extension degree and main results of this paper. The set of non-negative integers is denoted by $\omega$. For a subset $X$ of a linear space $L$ and $n \in \omega$, we define

$$
\langle X\rangle_{n}=\left\{\sum_{i=0}^{n} \lambda_{i} x_{i}: x_{i} \in X, \sum_{i=0}^{n} \lambda_{i}=1\right\} \subset L .
$$

Definition 1.1. Let $A$ be a closed subset of a space $X$. For a pair $(X, A)$, the extension degree $\mathrm{D}(A, X)$ is the minimal $n$ such that for any locally convex topological linear space $L$ any continuous map $f: A \rightarrow L$ extends to a continuous map from $X$ to $\langle f(A)\rangle_{n}$. If such an $n$ does not exist, we set $\mathrm{D}(A, X)=\infty$.

Let $L(X)$ be an algebraic linear space generated by $X$ and

$$
L_{n}(X)=\left\{\sum_{i=0}^{n} \lambda_{i} x_{i}: x_{i} \in X,\left|\lambda_{i}\right| \leq 1\right\}
$$

1991 Mathematics Subject Classification: 54C20, 46A16.

Key words and phrases: topological linear space, Dugundji extension theorem, Graev extension, extension degree, local convexity.

The author thanks K. Sakai for his help with Theorem 1.4 and valuable comments. He also thanks K. Kawamura for stimulating talks. 
for $n \in \omega$. In the sequel we deal with various topologies on $L(X)$ which make $L(X)$ a topological linear space containing $X$ as a topological subspace.

THEOREM 1.2. Suppose that for each $n \in \omega$ there exist a compact metrizable space $X_{n}$ and a closed set $P_{n} \subset X_{n}$ with $\mathrm{D}\left(P_{n}, X_{n}\right)>n$. Let $X=$ $\prod_{n \in \omega} X_{n}$ and $P=\prod_{n \in \omega} P_{n}$. Then $L(P)$ has the direct limit topology of a tower of compact metrizable spaces such that $L(P)$ is a topological linear space and the canonical embedding of $P$ in $L(P)$ does not extend continuously over $X$.

The free topological linear space $F L(X)$ on a space $X$ is defined categorically as usual, that is, $F L(X)$ is algebraically the same as $L(X)$ and for any topological linear space $L$ any continuous map $f: X \rightarrow L$ extends to a continuous linear map from $F L(X)$ to $L$. The free locally convex topological linear space $F L^{\text {lc }}(X)$ is defined similarly. $F L_{n}(X)$ and $F L_{n}^{\text {lc }}(X)$ are the subspaces of $F L(X)$ and $F L_{n}^{\mathrm{lc}}(X)$ respectively with the same base set $L_{n}(X)$.

THEOREM 1.3. Let $X$ be a separable space with a non-separable closed set $A$. Then there exists no continuous map from $X$ to $F L^{\mathrm{lc}}(A)$ which extends the canonical embedding of $A$ in $F L^{\mathrm{lc}}(A)$. Consequently, $\mathrm{D}(A, X)=\infty$.

It is known that there exists a compact separable space with a nonseparable closed set. For instance, let $\omega_{1}$ be the set of all countable ordinal numbers and $2=\{0,1\}$ the discrete space with two points. Then the product space $2^{\omega_{1}}$ is separable [11] and contains a closed subspace homeomorphic to the space $\omega_{1}+1$. Therefore, this theorem provides a simple proof of the well-known result of $[1,14]$ about closed convex sets in locally convex topological linear spaces: There exists a closed convex subset of a locally convex topological linear space which is not an absolute extensor for compact spaces.

Answering the author's question, K. Sakai proved the following theorem.

THEOREM 1.4. If a closed set $A$ is a neighborhood retract of a normal space $X$, then $\mathrm{D}(A, X) \leq 1$.

According to this theorem, to find a pair $(A, X)$ with $\mathrm{D}(A, X) \geq 2$ we must search for spaces which are not ANR's.

TheOREM 1.5. There exists a closed subset $P$ of $\mathbb{I}^{2}$ such that $\mathrm{D}\left(P, \mathbb{I}^{2}\right)=2$.

Remark 1.6. After the submission of the first version of this paper, R. Cauty [5] has constructed a $\sigma$-compact metrizable topological linear space $L$ which is not an absolute extensor for metrizable spaces. Though the construction of Theorem 1.2 of the present paper gives a less satisfactory result, it still seems to be interesting to decide whether there exists a closed subset $A$ of a finite-dimensional Euclidean space $\mathbb{E}$ with $\mathrm{D}(A, \mathbb{E}) \geq n$ for 
$n \geq 3$. Cauty's space $L$ is not an absolute extensor for compact metrizable spaces by [16, Corollary 3.7], that is, there exist a compact metrizable pair $(X, A)$ and a continuous map $\varphi$ from $A$ to $L$ which does not extend over $X$. For such $(X, A), \mathrm{D}(A, X)=\infty$ holds. To see this, suppose the existence of a continuous map $\psi: X \rightarrow\langle A\rangle_{n} \subset F L^{\text {lc }}(A)$ for some $n$ which extends the canonical embedding of $A$ in $F L^{\mathrm{lc}}(A)$. Since $\psi(X)$ is a compact subset of $\langle A\rangle_{n}$, there exists $m$ such that $\psi(X) \subset F L_{m}^{\text {lc }}(A)$. Since $F L_{m}(A)$ is compact, $F L_{m}(A)$ and $F L_{m}^{\text {lc }}(A)$ have the same topology. Thus $\psi$ can be regarded as a continuous map to $F L(A)$. The freeness of $F L(A)$ implies that $\varphi$ extends over $X$, which is a contradiction.

Since Cauty's proof uses Dranishnikov's space, it is unclear whether his result gives some information about the existence of a finite-dimensional pair $(X, A)$ with $\mathrm{D}(A, X) \geq 3$.

2. The Graev extensions of pseudo-metrics and proof of Theorem 1.2. We first introduce an extension of a continuous pseudo-metric on a space $X$ to $L(X)[15]$ and investigate its properties. Since this extension is defined analogously to the Graev metric for free abelian topological groups [12], we call it the Graev extension. (See also [2].)

Let $\varrho$ be a continuous pseudo-metric on a space $X$ and take a point $p^{*} \in$ $X$. We assume $X \subset L(X)$ and extend $\varrho$ to $X \cup\{0\}$ by $\varrho(x, 0)=1+\varrho\left(x, p^{*}\right)$. For $u, v \in L(X)$, define

$$
\begin{aligned}
& \bar{\varrho}(u, v)=\inf \left\{\sum_{i=0}^{m}\left|\lambda_{i}\right| \varrho\left(x_{i}, y_{i}\right):\right. \\
& \left.u=\sum_{i=0}^{m} \lambda_{i} x_{i}, v=\sum_{i=0}^{m} \lambda_{i} y_{i}, x_{i}, y_{i} \in X \cup\{0\}, \lambda_{i} \in \mathbb{R}\right\} .
\end{aligned}
$$

The above infimum is actually realized, that is, we have the following.

Proposition 2.1. For any continuous pseudo-metric $\varrho$ on $X$ and $u, v \in$ $L(X)$, there exist $x_{i}, y_{i} \in X \cup\{0\}$ and $\lambda_{i} \in \mathbb{R}$ such that $\bar{\varrho}(u, v)=\sum_{i=0}^{m}\left|\lambda_{i}\right| \times$ $\varrho\left(x_{i}, y_{i}\right), u=\sum_{i=0}^{m} \lambda_{i} x_{i}, v=\sum_{i=0}^{m} \lambda_{i} y_{i}$ and $x_{i}, y_{i} \in X$ appear in the reduced form of $u$ or $v$. In case $v=0$, the above holds with the additional condition that $x_{i} \neq 0$ for each $i$. Consequently, $\bar{\varrho}$ extends $\varrho$. In particular, if $\varrho$ is a metric, $\bar{\varrho}$ also becomes a metric and $L(X)$ becomes a normed linear space.

Here, $\sum_{i=0}^{m} \lambda_{i} x_{i}$ is the reduced form of $u$ if $u=\sum_{i=0}^{m} \lambda_{i} x_{i}, \lambda_{i} \neq 0$, $x_{i} \in X$ and $x_{i} \neq x_{j}$ for $i \neq j$. Though the above fact seems to be known [15], we have not been able to find its full proof in the literature. Since we use it frequently, we present the proof in Appendix for completeness. It is easy to check that $\|u\|=\bar{\varrho}(u, 0)$ is a seminorm on $L(X)$, i.e. $\|u\| \geq 0$, $\|u+v\| \leq\|u\|+\|v\|$ and $\|r u\|=|r|\|u\|$ for $r \in \mathbb{R}$. 
According to the universal property of $F L^{\text {lc }}(A), \mathrm{D}(A, X)$ is determined by extensions of the canonical embedding of $A$ in $F L^{\text {lc }}(A)$. On the other hand, Tkachenko [15] proved that the topology of $F L^{\text {lc }}(A)$ is determined by the Graev extensions of all continuous pseudo-metrics on $A$. As for free abelian topological groups [10, p. 362], this fact can be briefly proved using the Kakutani theorem [13, p. 68].

LEMma 2.2. Let $\varrho$ be a pseudo-metric on $X$. Then the canonical linear map $h: L(X) \rightarrow \mathbb{R}$, i.e. $h\left(\sum_{i=0}^{n} \lambda_{i} x_{i}\right)=\sum_{i=0}^{n} \lambda_{i}$ for $x_{i} \in X$ and $\lambda_{i} \in \mathbb{R}$, is continuous with respect to the Graev extension $\bar{\varrho}$. Consequently, $\langle X\rangle_{n}$ is closed in $L(X)$ with respect to $\bar{\varrho}$.

Proof. By Proposition 2.1, we can let $\bar{\varrho}\left(\sum_{i=0}^{m} \lambda_{i} x_{i}, 0\right)=\sum_{j=0}^{n}\left|\mu_{j}\right| \times$ $\varrho\left(y_{j}, z_{j}\right)$, where $\sum_{j=0}^{n} \mu_{j} y_{j}=\sum_{i=0}^{m} \lambda_{i} x_{i}$ and $\sum_{j=0}^{n} \mu_{j} z_{j}=0$ and $y_{j} \neq 0$ for each $j$. Let $F=\left\{j: z_{j}=0\right\}$. Then $\sum_{j \notin F} \mu_{j}=0$ and $\varrho\left(y_{j}, z_{j}\right) \geq 1$ for each $j \in F$. Hence,

$$
\left|\sum_{i=0}^{m} \lambda_{i}\right|=\left|\sum_{j=0}^{n} \mu_{j}\right|=\left|\sum_{j \in F} \mu_{j}\right| \leq \sum_{j \in F}\left|\mu_{j}\right| \varrho\left(y_{j}, z_{j}\right) \leq \bar{\varrho}\left(\sum_{i=0}^{m} \lambda_{i} x_{i}, 0\right),
$$

which implies the continuity of $h$.

The next lemma is a version of Graev's theorem [12, Theorem 4] about free (abelian) topological groups over compact spaces; almost the same statement has been proved by Borges [4, Theorem 2.2]. The proof can be done categorically using the following known fact: Let $X_{n} \subset X_{n+1}$ and $Y_{n} \subset Y_{n+1}(n \in \omega)$ be compact subsets of spaces $X$ and $Y$ respectively. Then $\lim _{\longrightarrow}\left\{X_{n} \times Y_{n}: n \in \omega\right\}=\lim _{\longrightarrow}\left\{X_{n}: n \in \omega\right\} \times \underline{\lim }\left\{Y_{n}: n \in \omega\right\}$. Hence, we omit the proof.

Lemma 2.3. Suppose that $Y_{n}(n \in \omega)$ are compact subsets of a topological linear space $L$ satisfying the following:

(1) $L=\bigcup_{n \in \omega} Y_{n}$ and $Y_{n} \subset Y_{n+1}(n \in \omega)$;

(2) For each $m \in \omega$, there exists $n \in \omega$ such that $Y_{m}+Y_{m} \subset Y_{n}$ and $r Y_{m} \subset Y_{n}$ for any $r \in \mathbb{R}$ with $|r| \leq m$.

Then the direct limit $\lim _{\longrightarrow}\left\{Y_{n}: n \in \omega\right\}$ regarded in the category of topological spaces (see e.g. [8]) is a topological linear space with the same algebraic operations as $L$.

LemMa 2.4. Let $A$ be a compact metrizable subset of $X$ and $\varrho$ a metric for $A$. Suppose that $\mathrm{D}(A, X)>n$. If $\varphi: X \rightarrow L(A)$ is a continuous extension of the canonical embedding of $A$ with respect to the metric $\bar{\varrho}$, then $\varphi(X)$ is not contained in $\langle A\rangle_{n} \cap L_{n}(A)$.

Proof. Let $L$ be a locally convex topological linear space. Then each continuous map $f: A \rightarrow L$ extends to a continuous linear map 
$\bar{f}: F L^{\text {lc }}(A) \rightarrow L$. Since $L(A)$ is locally convex by Proposition 2.1 , the topology of $F L^{\mathrm{lc}}(A)$ is finer than that of $L(A)$. Since $F L_{n}^{\mathrm{lc}}(A)$ is compact, the topology of $F L_{n}^{\text {lc }}(A)$ is the same as that of $L_{n}(A)$, i.e. $F L_{n}^{\text {lc }}(A)=L_{n}(A)$ as spaces. Suppose that $\varphi(X) \subset\langle A\rangle_{n} \cap L_{n}(A)$. Then $f$ extends to a continuous linear map $\bar{f} \varphi: X \rightarrow L$. By linearity of $\bar{f}$,

$$
\bar{f} \varphi(X) \subset \bar{f}\left(\langle A\rangle_{n} \cap L_{n}(A)\right) \subset \bar{f}\left(\langle A\rangle_{n}\right) \subset\langle f(A)\rangle_{n},
$$

which contradicts $\mathrm{D}(A, X)>n$.

Proof of Theorem 1.2. For each $n \in \omega$, let $\varrho_{n}$ be a metric on $P_{n}$ such that $\varrho_{n}(x, y) \leq 1$ for every $x, y \in P_{n}$. Define a metric $\varrho$ on the product space $P$ by $\varrho(x, y)=\sum_{n=0}^{\infty} 2^{-n} \varrho_{n}(x(n), y(n))$. For $k \in \omega$, let $\pi_{k}: P \rightarrow P_{k}$ be the projection and $i_{k}: X_{k} \rightarrow X$ be an injection defined by $i_{k}(x)(k)=x$ and $i_{k}(x)(n)=p_{n}^{*}$ for $n \neq k$, where $p_{n}^{*} \in P_{n}$. By Proposition 2.1, $\varrho$ induces a norm on $L(P)$. By Lemma 2.3, the direct limit $\underline{\lim }\left\{L_{m}(P): m \in \omega\right\}=L^{\sharp}$ is a topological linear space.

Suppose that the canonical embedding of $P$ in $L^{\sharp}$ extends to a continuous map $\varphi: X \rightarrow L^{\sharp}$. Let $U=\{u \in X: h \varphi(u)>0\}$, where $h$ is the canonical linear map in Lemma 2.2. Since $h^{-1}((0, \infty))$ is $\varrho$-open in $L(P)$ and hence open in $L^{\sharp}, U$ is an open neighborhood of $P$ in $X$. Define $\varphi^{\prime}: U \rightarrow$ $L^{\sharp}$ by $\varphi^{\prime}(u)=\varphi(u) / h(u)$. Since $L^{\sharp}$ has the direct limit topology, $\varphi(X)$ is contained in some $L_{k}(P)$ and hence $\varphi^{\prime}(U)$ is contained in some $\langle P\rangle_{k}$. Choose $k \in \omega$ large enough so that $i_{k}\left(X_{k}\right) \subset U$. Since $\pi_{k}$ extends to a continuous linear map $\bar{\pi}_{k}: L(P) \rightarrow L\left(P_{k}\right)$, we get a continuous map $\bar{\pi}_{k} \varphi^{\prime} i_{k}: X_{k} \rightarrow\left\langle P_{k}\right\rangle_{k} \cap L_{k}\left(P_{k}\right)$ which extends the canonical embedding of $P_{k}$. This contradicts $\mathrm{D}\left(P_{k}, X_{k}\right)>k$ by Lemma 2.4 .

The following is a corollary to Lemma 2.3 .

Corollary 2.5. Let $X$ be a compact space. Then the free topological linear space $F L(X)$ is the direct limit $\lim _{\longrightarrow}\left\{F L_{n}(X): n \in \omega\right\}$.

Proof. Since $F L_{n}(X)(n \in \omega)$ satisfy the properties in Lemma 2.3, the direct $\operatorname{limit} \lim \left\{F L_{n}(X): n \in \omega\right\}$ becomes a topological linear space. The direct limit topology is finer than the original one in general and hence the conclusion follows from the freeness of $F L(X)$.

R e mark 2.6. As we have already remarked, the Graev extensions of all continuous pseudo-metrics on $X$ determine the topology of the free locally convex topological linear space $F L^{\text {lc }}(X)$, which is similar to the case of free abelian topological groups. In addition, as is well known, Corollary 2.5 holds for free topological groups and free abelian topological groups. However, Corollary 2.5 does not hold for $F L^{\mathrm{lc}}(X)$. A simple example is given as follows. Let $X=\left\{c_{n}, c: n \in \omega\right\}$ be a non-trivial convergent sequence, i.e. $c_{m} \neq c_{n}$ for $m \neq n$ and $\lim _{n \rightarrow \infty} c_{n}=c$. Then $\lim _{n \rightarrow \infty} \sum_{k=1}^{n} n^{-1} c_{k}=c$ in 
$F L^{\text {lc }}(X)$ and consequently $\left\{\sum_{k=1}^{n} n^{-1} c_{k}, c: 1 \leq n \in \omega\right\}$ is a compact set in $F L^{\text {lc }}(X)$ which is not contained in any $F L_{n}^{\text {lc }}(X)$. Hence, $F L^{\text {lc }}(X)$ is not a direct limit of $F L_{n}^{\text {lc }}(X)$ 's. On the other hand, as mentioned in Remark 1.6, $F L_{n}(X)$ and $F L_{n}^{\text {lc }}(X)$ have the same topology for a compact space $X$.

\section{Proofs of Theorems $\mathbf{1 . 3}$ and $\mathbf{1 . 4}$}

Lemma 3.1. Let $X$ be a non-separable space and $X \subset Y \subset F L^{\mathrm{lc}}(X)$. Then $Y$ is not separable.

Pr o of. Suppose that $Y$ is separable, i.e. $Y$ has a countable dense subset $D$. Let $E$ be the set of points of $X$ which appear in the reduced forms of members of $D$. Then $E$ is obviously countable, hence is not dense in $X$. There exist a point $x \in X$ and a continuous pseudo-metric $\varrho$ on $X$ such that $\varrho(x, E) \geq 1$. By Proposition 2.1, $\varrho(x, D) \geq 1$, which contradicts the fact that $D$ is dense in $Y$.

Proof of Theorem 1.3. Suppose that the canonical embedding of $A$ in $F L^{\text {lc }}(A)$ extends to a continuous map $\varphi: X \rightarrow F L^{\text {lc }}(A)$. Then $\varphi(X)$ is separable and contains $A$, which contradicts Lemma 3.1.

Proof of Theorem 1.4. Let $U$ be an open neighborhood of $A$ in $X$ and $r: U \rightarrow A$. Choose an open neighborhood $V$ of $A$ such that $\bar{V} \subset U$ and let $h: X \rightarrow[0,1]$ be an Urysohn function such that $h(A)=\{1\}$ and $h(X \backslash V)=\{0\}$. Let $L$ be a topological linear space and $a \in A$. Then each continuous map $f: A \rightarrow L$ can be extended to $\varphi: X \rightarrow L$ by

$$
\varphi(x)= \begin{cases}h(x) f(r(x))+(1-h(x)) f(a) & \text { if } x \in V ; \\ f(a) & \text { otherwise. }\end{cases}
$$

Then $\varphi(X) \subset\langle f(A)\rangle_{1}$. Since $\varphi \mid U$ and $\varphi \mid X \backslash \bar{V}$ are continuous, $\varphi$ is continuous.

R e mark 3.2. We have applied a retraction in the proof of Theorem 1.4, but we cannot prove it just tracing the proof of the Dugundji theorem. In fact, let $f: A \rightarrow L$ be a continuous map from a closed subset $A$ of a separable metrizable space $X$ to a locally convex topological linear space $L$. The proof of the Dugundji extension theorem is based on a locally finite open cover. Hence, there exists a countable subset $C$ of $A$ such that the image of the extension of $f$ is contained in the union of $f(A)$ and the convex hull of $f(C)$. As the Baire category technique in the proof of Theorem 1.5 shows, we cannot replace the convex hull of $f(C)$ by $\langle f(C)\rangle_{1}$ even in a simple case of Theorem 1.4, e.g. a circle in the plane.

4. Proof of Theorem $\mathbf{1 . 5}$ and a related result. For the proof of Theorem 1.5, we recall a pseudo-circle in [3]. For a pseudo-arc and pseudocircle, we refer the reader to [6, Chap. 9]. A pseudo-arc is defined as the 
inverse limit of chains of closed disks, and a pseudo-circle is defined as the inverse limit of closed chains of closed disks. A pseudo-circle $P \subset \mathbb{I}^{2}\left(\subset \mathbb{R}^{2}\right)$ satisfies the following:

(1) $P$ is a continuum which divides $\mathbb{R}^{2}$ into two components;

(2) For any $\varepsilon>0$ there exists an auto-homeomorphism of $\mathbb{R}^{2}$ such that the distance of the image of the circle $\mathbb{S}^{1}$ and $P$ is less than $\varepsilon$;

(3) $P$ contains no non-trivial arc.

For a metric space $X=(X, \varrho)$, let $\mathcal{F}(X)$ be the hyperspace of finite subsets of $X$ with metric $\varrho(F, G)=\max \{\varrho(x, G), \varrho(y, F): x \in F, y \in G\}$ for $F, G \in \mathcal{F}(X)$, where $\varrho(x, G)=\min \{\varrho(x, y): y \in G\}$.

LEMMA 4.1. Let $X=(X, \varrho)$ be a metric space and $L(X)$ a normed linear space with the Graev extension $\bar{\varrho}$. Let $f: Y \rightarrow L(X)$ be a continuous map such that $f(Y) \subset\langle X\rangle_{k} \backslash\langle X\rangle_{k-1}$. Then $f$ induces a continuous map $g: Y \rightarrow \mathcal{F}(X)$ defined by $g(u)=\left\{x_{i}^{u}: 0 \leq i \leq k\right\}$ if $f(u)=\sum_{i=0}^{k} \lambda_{i}^{u} x_{i}^{u}$ is a reduced form. If $Y$ is path-connected and $X$ has no non-trivial arc, then $g$ is constant.

Proof. To see the continuity of $g$ at $u \in Y$, let $0<\varepsilon<1$ so that $\varrho\left(x_{i}^{u}, x_{j}^{u}\right)>2 \varepsilon$ if $i \neq j$ and let $M=\min \left\{\left|\lambda_{i}^{u}\right|: 0 \leq i \leq n\right\}$. Suppose that $\varrho(f(u), f(v))<M \varepsilon$. We recall that only $x_{i}^{u}$ 's, $x_{i}^{v}$ 's and 0 appear in the term which realizes the infimum concerning $\bar{\varrho}(f(u), f(v))$ by Proposition 2.1. Observe that the cardinalities of $g(u)$ and $g(v)$ are both $k+1$. Now, we fix $i$. Since $\varrho\left(x_{i}^{u}, x_{j}^{u}\right)>2 \varepsilon$ if $i \neq j$ and $\varrho\left(x_{i}^{u}, 0\right) \geq 1$, there exists a unique $j$ such that $\varrho\left(x_{i}^{u}, x_{j}^{v}\right)<\varepsilon$, which implies $\varrho(g(u), g(v))<\varepsilon$. Hence, $g$ is continuous at $u$.

Suppose that $g$ is not constant. Since $Y$ is path-connected, $\mathcal{F}(X)$ has a non-trivial arc in $X$. Hence, $X$ also has a non-trivial arc, which is a contradiction.

LEMMA 4.2. Let $h: \mathbb{I}^{2} \rightarrow \mathbb{R}^{2}$ be a continuous map which is the identity on $P$. Then any point in the bounded component of $\mathbb{R}^{2} \backslash P$ belongs to $h\left(\mathbb{I}^{2}\right)$.

Proof. Suppose that there exists a point $q$ in the bounded open component of $\mathbb{R}^{2} \backslash P$ which does not belong to $h\left(\mathbb{I}^{2}\right)$. Take $\varepsilon>0$ so that $\varrho(q, P)>2 \varepsilon$. Since $h$ is uniformly continuous, there exists $0<\delta<\varepsilon / 2$ such that $\varrho(x, y)<\delta$ implies $\varrho(h(x), h(y))<\varepsilon / 2$. By the property (2) of a pseudo-circle, there exists a continuous map $f: \mathbb{S}^{1} \rightarrow \mathbb{I}^{2} \backslash\{q\}$ such that $\varrho(f(u), P)<\delta$ for any $u \in \mathbb{S}^{1}$ and $f$ is not null-homotopic. For each $u \in \mathbb{S}^{1}$, take $x \in P$ so that $\varrho(f(u), x)<\delta$. Then $\varrho(f(u), h f(u)) \leq$ $\varrho(f(u), x)+\varrho(x, h f(u))=\varrho(f(u), x)+\varrho(h(x), h f(u))<\varepsilon / 2+\varepsilon / 2=\varepsilon$. On the other hand, $\varrho(f(u), q)>3 \varepsilon / 2$. Therefore, $h f: \mathbb{S}^{1} \rightarrow \mathbb{R}^{2} \backslash\{q\}$ is homotopic to $f$. On the other hand, $h f$ is null-homotopic, since $\mathbb{I}^{2}$ is contractible. This is a contradiction. 
Proof of Theorem 1.5. We denote the Euclidean metric for $\mathbb{R}^{2}$ by $\varrho$ and let $\|x\|=\varrho(x, 0)$. There is a natural linear map $\psi: L(P) \rightarrow \mathbb{R}^{2}$, i.e. $\psi\left(\sum_{i=0}^{k} \lambda_{i} x_{i}\right)=\sum_{i=0}^{k} \lambda_{i} x_{i}$ for $x_{i} \in P$ and $k \in \omega$. First, we show that $\psi$ is continuous. To avoid ambiguity, we denote the restriction of $\varrho$ to $P$ by $\varrho_{P}$. In the definition of $\varrho_{P}, p^{*} \in P$ has been chosen and $\varrho_{P}\left(0, p^{*}\right)=1$. Note that the 0 in $L(P)$ is not in $P$, but the 0 in $\mathbb{R}^{2}$ may belong to $P$. Therefore, $\varrho\left(0, p^{*}\right)=\left\|p^{*}\right\|$ may not be equal to 1 . Take $N \geq 1$ so that $\varrho\left(0, p^{*}\right) \leq N$. Suppose that $\bar{\varrho}_{P}(u, v)<\varepsilon / N$. Then $\sum_{i=0}^{m}\left|\lambda_{i}\right| \varrho_{P}\left(x_{i}, y_{i}\right)<$ $\varepsilon / N, u=\sum_{i=0}^{m} \lambda_{i} x_{i}, v=\sum_{i=0}^{m} \lambda_{i} y_{i}$, for some $x_{i}, y_{i} \in X \cup\{0\}$ and $\lambda_{i} \in \mathbb{R}$. Now,

$$
\begin{aligned}
\|\psi(u)-\psi(v)\| & =\left\|\sum_{i=0}^{m} \lambda_{i} x_{i}-\sum_{i=0}^{m} \lambda_{i} y_{i}\right\| \\
& \leq \sum_{i=0}^{m}\left|\lambda_{i}\right|\left\|x_{i}-y_{i}\right\| \leq \sum_{i=0}^{m}\left|\lambda_{i}\right| N \varrho_{P}\left(x_{i}, y_{i}\right)<\varepsilon,
\end{aligned}
$$

which shows the continuity of $\psi$.

To obtain a contradiction, suppose that the canonical embedding of $P$ in $L(P)$ extends to a $\bar{\varrho}_{P}$-continuous map $\varphi: \mathbb{I}^{2} \rightarrow\langle P\rangle_{1} \subset L(P)$. We are interested in parts of $\varphi\left(\mathbb{I}^{2}\right)$ which are not in $P$. We define $\overline{p q}=\{t p+(1-t) q$ : $0 \leq t \leq 1\} \subset L(P)$ for distinct points $p, q \in P$. Since $P$ is closed by Lemma $2.2, \varphi^{-1}(L(P) \backslash P)$ is a countable union of connected open subsets of $\mathbb{I}^{2}$. Let $O$ be one of such open components. Then by the assumption and Lemma 4.1 there exist distinct $p, q \in P$ such that $\varphi(O) \subset \overline{p q}$. Therefore, there exists a countable family $\mathcal{C}$ consisting of pairs of members of $P$ such that $\varphi\left(\mathbb{I}^{2}\right) \backslash P \subset \bigcup\{\overline{p q}:\{p, q\} \in \mathcal{C}\}$. Now, $\psi \varphi\left(\mathbb{I}^{2}\right) \subset P \cup \bigcup\{\psi(\overline{p q}):\{p, q\} \in$ $\mathcal{C}$ \}. Since $\psi$ is linear, each $\psi(\overline{p q})$ is a nowhere dense closed set in $\mathbb{R}^{2}$. By the Baire category theorem, there exists a point $p$ in the bounded open component of $\mathbb{R}^{2} \backslash P$ such that $p$ does not belong to $\psi \varphi\left(\mathbb{I}^{2}\right)$. Since $\psi \varphi$ is the identity on $P$, we get a contradiction by Lemma 4.2.

In the proof of the Dugundji extension theorem [7], one takes a locally finite refinement. The multiplicity of this refinement depends on the dimension of $\mathbb{I}^{2} \backslash P$, i.e. $\mathrm{D}\left(P, \mathbb{I}^{2}\right) \leq 2$, which now implies $\mathrm{D}\left(P, \mathbb{I}^{2}\right)=2$.

In the proof of Theorem 1.5, we show $\mathrm{D}\left(P, \mathbb{I}^{2}\right)=2$ for a pseudo-circle $P$. Now, we show this also holds for a pseudo-arc $P$. A pseudo-arc $P \subset \mathbb{I}^{2}$ $\left(\subset \mathbb{R}^{2}\right)$ satisfies the following:

(1) $P$ is a degenerate continuum;

(2) For any points $p, q \in P$ and any $\varepsilon>0$ there exists an arc from $p$ to $q$ such that the distance of the arc and $P$ is less than $\varepsilon$;

(3) $P$ contains no non-trivial arc.

Theorem 4.3. For a pseudo-arc $P$ in $\mathbb{I}^{2}, \mathrm{D}\left(P, \mathbb{I}^{2}\right)=2$. 
To prove this theorem, we use words of infinite length and refer the reader to [9]. Since we use the listed properties of a pseudo-arc, the proof is also valid for a pseudo-circle. Let $\mathcal{L}$ be a set of ordered pairs $(p, q)$ for distinct points $p, q \in P$ such that either $(p, q) \in \mathcal{L}$ or $(q, p) \in \mathcal{L}$ and only one of them holds. We take $\mathcal{L}$ as the set of letters. In the remaining part of this section, we assume that $L(P)$ is endowed with the Graev extension $\bar{\varrho}$ of the Euclidean metric $\varrho$ on $P$. For a continuous map $f:[a, b] \rightarrow\langle P\rangle_{1} \subset L(P)$, let $W^{f}: \overline{W^{f}} \rightarrow \mathcal{L}$ be the $\sigma$-word defined as follows:

$$
\begin{aligned}
\overline{W^{f}}=\{(c, d): a \leq c<d \leq b, f(c), f(d) & \in P, \\
f((c, d)) & \subset \overline{p q} \text { for some }(p, q) \in \mathcal{L}\},
\end{aligned}
$$

and the ordering of $\overline{W^{f}}$ is induced from the ordering on $[0,1] ; W^{f}((c, d))=$ $(f(c), f(d))$ if $(f(c), f(d)) \in \mathcal{L} ; W^{f}((c, d))=(f(d), f(c))^{-1}$ otherwise. First we state an easy lemma about a free product.

LEMMA 4.4. Let $G$ be a group and $\langle F\rangle$ the free group generated by $F=$ $\left\{\alpha_{0}, \ldots, \alpha_{m}\right\}$. For $0 \leq i \leq n$, let $g_{i}$ be elements of the free product $\langle F\rangle * G$ such that $g_{i} \in G$ or $g_{i}=\alpha^{\varepsilon}$ for some $\alpha \in F$ and $\varepsilon= \pm 1$. If $g_{0} \ldots g_{n}=e$, then there exists an increasing sequence $0 \leq m_{0}<\ldots<m_{k} \leq n$ with the following properties:

(1) For each $0 \leq i<k$, either $g_{j} \in G$ for every $m_{i} \leq j<m_{i+1}$ or $g_{m_{i}}=g_{m_{i+1}-1}^{-1} \in\langle F\rangle$;

(2) $\prod_{i \in I} g_{m_{i}} \ldots g_{m_{i+1}-1}=e$, where $i \in I$ if and only if $g_{j} \in G$ for every $m_{i} \leq j<m_{i+1}$.

Proof. Fix a reduction of $g_{0} \ldots g_{n}$ to the empty word. Of course, each $g_{j} \in\left\{\alpha, \alpha^{\varepsilon}: \alpha \in F\right\}$ is canceled in the reduction process. Make pairs which cancel together; then pairings are not tangled. Hence, picking outermost pairs, we easily get the desired sequence.

LEMMA 4.5. Let $f:[0,1] \rightarrow\langle P\rangle_{1}$ be a path such that $f(0), f(1) \in P$ and $f(0) \neq f(1)$. Then $W^{f} \neq e$, where $e$ is the unit of $X_{\mathcal{L}} \mathbb{Z}$.

Proof. We deform $f$ to a path $g$ so that

(1) $g((a, b))=f(a)$ if $f(a)=f(b) \in P$ and $f((a, b)) \subset \overline{f(a) p}$ for some $p \in P$ with $p \neq f(a)$ and

(2) $g(x)=f(x)$ otherwise.

Then $W^{g}=W^{f}$ and hence we may assume that $f$ shares the same property as $g$, i.e. $f(x) \notin P$ corresponds to some $W^{f}((a, b))$ by Lemma 4.1. To argue by contradiction, suppose $W^{f}=e$. For a finite subset $F=\left\{\alpha_{0}, \ldots, \alpha_{k}\right\}$ of $\operatorname{Im}\left(W^{f}\right)$, we can take $a_{i}(0 \leq i \leq m)$ so that

(1) $a_{0}=0<a_{1}<\ldots<a_{m-1}<a_{m}=1$ and $f\left(a_{i}\right) \in P$;

(2) $W^{f}=W^{f \mid\left(a_{0}, a_{1}\right)} \ldots W^{f \mid\left(a_{m-1}, a_{m}\right)}$; 
(3) $W^{f \mid\left(a_{i}, a_{i+1}\right)} \in\left\{\alpha, \alpha^{\varepsilon}: \alpha \in F, \varepsilon= \pm 1\right\} \cup \mathcal{W}(\mathcal{L} \backslash F)$, where $\mathcal{W}(\mathcal{L} \backslash F)$ is the set of $\sigma$-words consisting of letters in $\mathcal{L} \backslash F$.

By Lemma 4.4, we get a subsequence $b_{j}(0 \leq j \leq n)$ of $a_{i}(0 \leq i \leq m)$ and $J \subset\{0, \ldots, n\}$ so that $b_{0}=0, b_{n}=1, f\left(b_{j}\right)=f\left(b_{j+1}\right)$ if $j \notin J$, and $\prod_{j \in J} W^{f \mid\left(b_{j}, b_{j+1}\right)}=e$, where $j \in J$ iff $W^{f \mid\left(b_{j}, b_{j+1}\right)} \in \mathcal{W}(\mathcal{L} \backslash F)$ and the product is performed according to the ordering of $[0,1]$. Define $h:[0,1] \rightarrow$ $\langle P\rangle_{1}$ by $h(x)=f\left(b_{j}\right)$ for $b_{j} \leq x \leq b_{j+1}$ if $W^{f \mid\left(b_{j}, b_{j+1}\right)} \notin \mathcal{W}(\mathcal{L} \backslash F)$; and $h(x)=f(x)$ otherwise. Then $h$ is a path from $f(0)$ to $f(1), W^{h} \in \mathcal{W}(\mathcal{L} \backslash F)$ and $W^{h}=\prod_{j \in J} W^{f \mid\left(b_{j}, b_{j+1}\right)}=e$.

Let $F_{n} \subset F_{n+1}$ be finite subsets of $\mathcal{L}$ such that $\operatorname{Im}\left(W^{f}\right)=\bigcup_{n=0}^{\infty} F_{n}$. We define $f_{n}$ inductively using $F_{n}$ and the above process of passing from $f$ to $h$. If $f_{n}(x) \neq f(x)$, then for some $\varepsilon$-neighborhood $U_{\varepsilon}(x)$ of $x, f_{n}\left(U_{\varepsilon}(x)\right)=$ $\{f(x)\}$ and moreover $f_{m}$ is constant on this neighborhood for $m \geq n$. Suppose that $f_{n}(x)$ is not eventually constant. There exists a sequence of closed intervals $\left[a_{n}, b_{n}\right](n \geq m)$ for some $m$ such that $a_{n+1} \leq a_{n}<x<b_{n} \leq b_{n+1}$ and $f_{n}\left(a_{n}\right)=f\left(a_{n}\right)=f\left(b_{n}\right)=f_{n}\left(b_{n}\right)$. Hence, $\lim _{n \rightarrow \infty} f_{n}(x)$ exists and the continuity of $\lim _{n \rightarrow \infty} f_{n}(x)$ at such an $x$ is clear. On the other hand, if $f_{n}(x)=f(x)$ for any $n$, then the continuity of the limit at $x$ follows from that of $f$. Now, $\lim _{n \rightarrow \infty} f_{n}(x)$ is a non-trivial path in $P$, which is a contradiction.

LEMMA 4.6. If $f:[0,1] \rightarrow\langle P\rangle_{1}$ is a loop and null-homotopic relative to $\{0,1\}$, then $W^{f}=e$.

Pr o of. Suppose $W^{f} \neq e$. Then there exists a finite subset $F$ of $\mathcal{L}$ with $\left(W^{f}\right)_{F} \neq e$. Deforming $f$ as in the first step of the proof of Lemma 4.5, we get a loop homotopic to $f$ relative to $\{0,1\}$. Hence, we may assume the existence of subintervals $\left(a_{i}, b_{i}\right)(0 \leq i \leq m)$ of $[0,1]$ such that $b_{i} \leq a_{i+1}$, $f\left(a_{i}\right), f\left(b_{i}\right) \in P$ and $\bigcup_{i=0}^{m}\left(a_{i}, b_{i}\right)=\bigcup_{(p, q) \in F} f^{-1} \overline{p q}$. Let $H:[0,1] \times[0,1] \rightarrow$ $\langle P\rangle_{1}$ be the homotopy from $f$ to the constant $f(0)$, i.e. $H(s, 0)=f(0)$, $H(s, 1)=f(s), H(0, t)=H(1, t)=f(0)$. Let $O$ be an open component of $\bigcup_{(p, q) \in F} H^{-1} \overline{p q}$ which contains some $\left(a_{i}, b_{i}\right) \times\{1\}$. Then there exists a unique $(p, q) \in F$ such that $H(O) \subset \overline{p q}$ by Lemma 4.1 and hence $H(x)=p$ or $q$ for $x \in \bar{O} \backslash O$. Let $i_{0}<\ldots<i_{k}$ be all the $i$ 's such that $\left(a_{i}, b_{i}\right) \times\{1\} \subset O$. Then $H\left(b_{i_{j}}, 1\right)=H\left(a_{i_{j+1}}, 1\right)$ for $0 \leq j \leq k-1$ and $H\left(b_{i_{k}}, 1\right)=H\left(a_{i_{0}}, 1\right)$. Therefore, $W^{\varphi h \mid\left(a_{i_{0}}, b_{i_{0}}\right)} \ldots W^{\varphi h \mid\left(a_{i_{k}}, b_{i_{k}}\right)}=e$. Let $O$ and $O^{\prime}$ be distinct open components of $\bigcup_{(p, q) \in F} H^{-1} \overline{p q}$. Then $O \cap[0,1] \times\{1\}$ and $O^{\prime} \cap[0,1] \times\{1\}$ are never nested. Considering the innermost components with respect to this situation on $[0,1] \times\{1\}$, we see that $W^{f \mid\left(a_{0}, b_{0}\right)} \ldots W^{f \mid\left(a_{m}, b_{m}\right)}=e$. But $W^{f \mid\left(a_{0}, b_{0}\right)} \ldots W^{f \mid\left(a_{m}, b_{m}\right)}=\left(W^{f}\right)_{F} \neq e$, which is a contradiction.

Proof of Theorem 4.3. As for a pseudo-circle, $\mathrm{D}\left(P, \mathbb{I}^{2}\right) \leq 2$ clearly. To show $\mathrm{D}\left(P, \mathbb{I}^{2}\right) \geq 2$ by contradiction, suppose that a continuous map $\varphi$ : 
$\mathbb{I}^{2} \rightarrow\langle P\rangle_{1}$ extends the canonical embedding of $P$. Take a path $f:[0,1] \rightarrow \mathbb{I}^{2}$ such that $f(0), f(1) \in P$ and $f(0) \neq f(1)$. Since $W^{\varphi f} \neq e$ by Lemma 4.5, there exists a finite subset $F$ of $\mathcal{L}$ such that $\left(W^{\varphi f}\right)_{F} \neq e$. Take $\varepsilon>0$ so that $\bar{\varrho}(P, p / 2+q / 2)>\varepsilon$ for any $(p, q) \in F$ and then a path $g:[0,1] \rightarrow \mathbb{I}^{2}$ so that $g(0)=f(1), g(1)=f(0)$ and $\bar{\varrho}(P, \varphi g(t))<\varepsilon$ for any $0 \leq t \leq 1$. Define $h$ by $h(t)=f(2 t)$ for $0 \leq t \leq 1 / 2$ and $h(t)=g(2 t-1)$ for $1 / 2 \leq t \leq 1$. Since $\varphi h$ goes through $\mathbb{I}^{2}, \varphi h$ is a loop null-homotopic relative to $\{0,1\}$. On the other hand, $W^{\varphi h} \neq e$, which contradicts Lemma 4.6.

Appendix. Here, we give a proof of Proposition 2.1. We introduce some notions and state easy facts for them.

Let $\left\langle\lambda_{i}: i \in I\right\rangle$ and $\left\langle\mu_{j}: j \in J\right\rangle$ be indexed finite families of non-zero reals. We call $\left\langle\mu_{j}: j \in J\right\rangle$ a refinement of $\left\langle\lambda_{i}: i \in I\right\rangle$ if there exist $J_{i}(i \in I)$ such that

(1) $J=\bigcup_{i \in I} J_{i}, J_{i} \cap J_{j}=\emptyset(i \neq j)$;

(2) $\lambda_{i}=\sum_{j \in J_{i}} \mu_{j}$

(3) $\lambda_{i}>0$ implies $\mu_{j}>0$ for all $j \in J_{i}$, and $\lambda_{i}<0$ implies $\mu_{j}<0$ for all $j \in J_{i}$.

Let $\sum_{i \in I} \lambda_{i}=0$. A refinement $\left\langle\mu_{j}: j \in J\right\rangle$ of $\left\langle\lambda_{i}: i \in I\right\rangle$ is called a good refinement if there exists a set $P$ of ordered pairs of members of $J$ such that $2|P|=|J|, \mu_{p_{0}}+\mu_{p_{1}}=0$ for $p=\left\langle p_{0}, p_{1}\right\rangle$, and $J=\left\{p_{0}, p_{1}: p \in P\right\}$.

The following are straightforward:

(1) For a refinement $\left\langle\mu_{j}: j \in J\right\rangle$ of $\left\langle\lambda_{i}: i \in I\right\rangle, \sum_{i \in I} \lambda_{i}=\sum_{j \in J} \mu_{j}$.

(2) If $\sum_{i \in I} \lambda_{i}=0$, then $\left\langle\lambda_{i}: i \in I\right\rangle$ has a good refinement.

(3) Let $\left\langle\mu_{j}: j \in J\right\rangle$ be a refinement of $\left\langle\lambda_{i}: i \in I\right\rangle$ and $u_{j}=x_{i}, v_{j}=y_{i}$ for $j \in J_{i}$. Then $\sum_{i \in I}\left|\lambda_{i}\right| \varrho\left(x_{i}, y_{i}\right)=\sum_{j \in J}\left|\mu_{j}\right| \varrho\left(u_{j}, v_{j}\right)$.

Proof of Proposition 2.1. We can write $u=\sum_{i=0}^{m} \lambda_{i} x_{i}$ and $v=$ $\sum_{i=0}^{m} \lambda_{i} y_{i}$ with $\lambda_{i} \neq 0$ and $x_{i}, y_{i} \in X \cup\{0\}$. Suppose that $x \in X$ appears in the reduced form of neither $u$ nor $v$, but $x$ is one of $x_{i}$ 's. Put $F=\{0 \leq$ $\left.i \leq m: x_{i}=x\right\}$; then $\sum_{i \in F} \lambda_{i}=0$. Take a good refinement of $\left\langle\lambda_{i}: i \in F\right\rangle$, say $\left\langle\mu_{j}: j \in J\right\rangle$. Using the above notation, let $v_{j}=y_{i}$ for $j \in J_{i}$ and $P^{\prime}=\left\{p \in P: v_{p_{0}} \neq x\right.$ or $\left.v_{p_{1}} \neq x\right\}$. In addition, for each $p \in P^{\prime}$, let $u_{p_{0}}=u_{p_{1}}=v_{p_{0}}$ if $v_{p_{0}} \neq x$, and let $u_{p_{0}}=u_{p_{1}}=v_{p_{1}}$ otherwise. Then

$$
\begin{aligned}
u & =\sum_{i \notin F} \lambda_{i} x_{i}=\sum_{i \notin F} \lambda_{i} x_{i}+\sum_{p \in P^{\prime}}\left(\mu_{p_{0}} u_{p_{0}}+\mu_{p_{1}} u_{p_{1}}\right), \\
v & =\sum_{i \notin F} \lambda_{i} y_{i}+\sum_{i \in F} \sum_{j \in J_{i}} \mu_{j} y_{i}=\sum_{i \notin F} \lambda_{i} y_{i}+\sum_{p \in P^{\prime}}\left(\mu_{p_{0}} v_{p_{0}}+\mu_{p_{1}} v_{p_{1}}\right) .
\end{aligned}
$$


Since

$$
\begin{aligned}
& \sum_{p \in P^{\prime}}\left(\left|\mu_{p_{0}}\right| \varrho\left(u_{p_{0}}, v_{p_{0}}\right)+\left|\mu_{p_{1}}\right| \varrho\left(u_{p_{1}}, v_{p_{1}}\right)\right) \\
& =\sum_{p \in P^{\prime}}\left|\mu_{p_{0}}\right| \varrho\left(v_{p_{0}}, v_{p_{1}}\right) \leq \sum_{p \in P^{\prime}}\left|\mu_{p_{0}}\right|\left(\varrho\left(x, v_{p_{0}}\right)+\varrho\left(x, v_{p_{1}}\right)\right) \\
& =\sum_{p \in P^{\prime}}\left(\left|\mu_{p_{0}}\right| \varrho\left(x, v_{p_{0}}\right)+\left|\mu_{p_{1}}\right| \varrho\left(x, v_{p_{1}}\right)\right) \\
& =\sum_{i \in F} \sum_{j \in J_{i}}\left|\mu_{j}\right| \varrho\left(x, y_{i}\right)=\sum_{i=0}^{m}\left|\lambda_{i}\right| \varrho\left(x_{i}, y_{i}\right),
\end{aligned}
$$

it follows that

$$
\sum_{i \notin F}\left|\lambda_{i}\right| \varrho\left(x_{i}, y_{i}\right)+\sum_{p \in P^{\prime}}\left(\left|\mu_{p_{0}}\right| \varrho\left(u_{p_{0}}, v_{p_{0}}\right)+\left|\mu_{p_{1}}\right| \varrho\left(u_{p_{1}}, v_{p_{1}}\right)\right) \leq \sum_{i=0}^{m}\left|\lambda_{i}\right| \varrho\left(x_{i}, y_{i}\right) \text {. }
$$

Thus we can remove $x$ from a representation of $u$ without adding new $x$ 's in a representation of $v$ nor increasing the distance. By repeating the same argument for $v$, we can remove $x$ from both representations of $u$ and $v$. By this transformation, we may restrict the range of $x_{i}, y_{i} \in X$ to the ones appearing in the reduced form of $u$ or $v$ for the calculation of $\bar{\varrho}(u, v)$.

Next, we show that we may also restrict the range of coefficients. Since $\bar{\varrho}(u, v)=\bar{\varrho}(u-v, 0)$, we may assume $v=0$ to get the conclusion. Let $u=\sum_{i=0}^{m} \lambda_{i} x_{i}$ be the reduced form, $S=\left\{x_{i}: 0 \leq i \leq m\right\} \cup\{0\}$ and $u=\sum_{j=0}^{n} \mu_{j} y_{j}, 0=\sum_{j=0}^{n} \mu_{j} z_{j}$, where $\mu_{j} \neq 0$ and $y_{j}, z_{j} \in S$. Fix $i$, and let $F=\left\{0 \leq j \leq n: y_{j}=x_{i}\right\}$. Suppose $\lambda_{i}>0$ but $\mu_{j_{0}}<0$ for some $j_{0} \in F$. By taking a refinement, we may assume there exists a subset $G$ of $F$ such that $\mu_{j}>0$ for $j \in G$ and $\mu_{j_{0}}+\sum_{j \in G} \mu_{j}=0$.

In case $z_{j_{0}}=0$, let $u_{j}=y_{j}$ for $j \notin G$ and $u_{j}=0$ for $j \in G$. Then $u=\sum_{j \neq j_{0}} \mu_{j} u_{j}, 0=\sum_{j \neq j_{0}} \mu_{j} z_{j}$ and

$$
\sum_{j \neq j_{0}}\left|\mu_{j}\right| \varrho\left(u_{j}, z_{j}\right) \leq \sum_{j=0}^{n}\left|\mu_{i}\right| \varrho\left(y_{j}, z_{j}\right),
$$

since

$$
\begin{aligned}
\sum_{j \in G}\left|\mu_{j}\right| \varrho\left(0, z_{j}\right) & \leq \sum_{j \in G}\left|\mu_{j}\right|\left(\varrho\left(x_{i}, 0\right)+\varrho\left(x_{i}, z_{j}\right)\right) \\
& =\left|\mu_{j_{0}}\right| \varrho\left(x_{i}, 0\right)+\sum_{j \in G}\left|\mu_{i}\right| \varrho\left(x_{i}, z_{j}\right) .
\end{aligned}
$$

In case $z_{j_{0}} \neq 0$, by the same argument, we can take $H \subset\{0, \ldots, n\}$ such that $z_{j}=z_{j_{0}}$ and $\mu_{j}>0$ for $j \in H$ and $\mu_{j_{0}}+\sum_{j \in H} \mu_{j}=0$. By taking a refinement, we may assume the existence of a bijection $e: G \rightarrow H$ such that 
$\mu_{j}=\mu_{e(j)}$ for $j \in G$ and $e(j)=j$ for $j \in G \cap H$. Then

$$
\begin{aligned}
u & =\sum_{j \notin G \cup H \cup\left\{j_{0}\right\}} \mu_{j} y_{j}+\sum_{j \in G \backslash H} \mu_{e(j)} y_{e(j)}, \\
0 & =\sum_{j \notin G \cup H \cup\left\{j_{0}\right\}} \mu_{j} z_{j}+\sum_{j \in G \backslash H} \mu_{j} z_{j},
\end{aligned}
$$

and

$$
\sum_{j \notin G \cup H \cup\left\{j_{0}\right\}}\left|\mu_{j}\right| \varrho\left(y_{j}, z_{j}\right)+\sum_{j \in G \backslash H}\left|\mu_{j}\right| \varrho\left(y_{e(j)}, z_{j}\right) \leq \sum_{j=0}^{n}\left|\mu_{j}\right| \varrho\left(y_{j}, z_{j}\right)
$$

by the inequality

$$
\begin{aligned}
\left|\mu_{j}\right| \varrho\left(y_{e(j)}, z_{j}\right) & \leq\left|\mu_{j}\right|\left(\varrho\left(y_{e(j)}, z_{j_{0}}\right)+\varrho\left(y_{j_{0}}, z_{j_{0}}\right)+\varrho\left(y_{j_{0}}, z_{j}\right)\right) \\
& \leq\left|\mu_{e(j)}\right| \varrho\left(y_{e(j)}, z_{e(j)}\right)+\left|\mu_{j_{0}}\right| \varrho\left(y_{j_{0}}, z_{j_{0}}\right)+\left|\mu_{j}\right| \varrho\left(y_{j}, z_{j}\right) .
\end{aligned}
$$

Thus we may assume that if $\lambda_{i}>0$ then $0<\mu_{j} \leq \lambda_{i}$ for all $j \in F$ and similarly if $\lambda_{i}<0$ then $\lambda_{i} \leq \mu_{j}<0$ for all $j \in F$. Applying this transformation for every $0 \leq i \leq m$, we may assume $\left|\mu_{j}\right| \leq M=\max \left\{\left|\lambda_{i}\right|: 0 \leq i \leq m\right\}$ for all $j$. Let $n=(m+2)^{2}$. We conclude $\varrho(u, 0)=\inf \left\{\sum_{j=0}^{n}\left|\mu_{j}\right| \varrho\left(y_{j}, z_{j}\right): u=\right.$ $\left.\sum_{j=0}^{n} \mu_{j} y_{j}, 0=\sum_{j=0}^{n} \mu_{j} z_{j},\left|\mu_{j}\right| \leq M, y_{j}, z_{j} \in S\right\}$. If we regard $\sum_{j=0}^{n}\left|\mu_{j}\right| \times$ $\varrho\left(y_{j}, z_{j}\right)$ as a function with variables $y_{j}, z_{j}, \mu_{j}(0 \leq j \leq n)$, its domain can be taken as a compact set as above. Therefore, we get the first statement of Proposition 2.1.

In case $v=0$, let $u=\sum_{i=0}^{m} \lambda_{i} x_{i}$ and $v=\sum_{i=0}^{m} \lambda_{i} y_{i}$ with $\lambda_{i} \neq 0$ and $x_{i}, y_{i} \in X \cup\{0\}$. Since $\sum_{i=0}^{m} \lambda_{i}=0$, we can take a good refinement for $\left\{\lambda_{i}: 0 \leq i \leq m\right\}$. Using this, we can remove $x_{i}=0$ without increasing the distance. Hence, we get the second statement. By the first statement, it is easy to see that $\bar{\varrho}$ extends $\varrho$. If $\varrho$ is a metric, $\bar{\varrho}$ also becomes a metric, because the infimum is realized.

There are many ways of embedding a metric space in a normed linear space isometrically. The next proposition shows that the embedding of Proposition 2.1, i.e. the one using the Graev extension, is canonical among them.

Proposition A.1. Let $(X, \varrho)$ be a metric space and regard $L(X)$ as a normed space with norm $\|u\|=\bar{\varrho}(u, 0)$. Suppose that $e: X \rightarrow L$ is an isometric embedding of $X$ in a normed linear space $L$. Then the canonical linear map $\varphi: L(X) \rightarrow L$, i.e. $\varphi(x)=e(x)$ for each $x \in X$, becomes continuous.

Proof. Take $M \geq 1$ so that $\left\|e\left(p^{*}\right)\right\| \leq M$. By Proposition 2.1, we can set, for $u \in L(X), \bar{\varrho}(u, 0)=\sum_{i=0}^{n}\left|\lambda_{i}\right| \varrho\left(x_{i}, y_{i}\right)$ where $u=\sum_{i=0}^{n} \lambda_{i} x_{i}$, $0=\sum_{i=0}^{n} \lambda_{i} y_{i}, x_{i} \in X, y_{i} \in X \cup\{0\}$. If $y_{i} \neq 0$, then $\left\|e\left(x_{i}\right)-e\left(y_{i}\right)\right\|=$ 


$$
\begin{aligned}
& \varrho\left(x_{i}, y_{i}\right) \leq M \varrho\left(x_{i}, y_{i}\right) . \text { Otherwise } \\
& \begin{aligned}
\left\|e\left(x_{i}\right)-e\left(y_{i}\right)\right\| & =\left\|e\left(x_{i}\right)\right\| \leq\left\|e\left(x_{i}\right)-e\left(p^{*}\right)\right\|+\left\|e\left(p^{*}\right)\right\| \leq \varrho\left(x_{i}, p^{*}\right)+M \\
& \leq M\left(\varrho\left(x_{i}, p^{*}\right)+1\right)=M \varrho\left(x_{i}, 0\right)=M \varrho\left(x_{i}, y_{i}\right) .
\end{aligned}
\end{aligned}
$$

Therefore, $\|\varphi(u)\| \leq \sum_{i=0}^{n}\left|\lambda_{i}\right| M \varrho\left(x_{i}, y_{i}\right)=M \bar{\varrho}(u, 0)=M\|u\|$, which implies the continuity of $\varphi$.

\section{REFERENCES}

[1] R. F. Arens, Extension of functions on fully normal spaces, Pacific J. Math. 2 (1952), 11-22.

[2] R. F. Arens and J. Eells, Jr., On embedding uniform topological spaces, ibid. 6 (1956), 397-403.

[3] R. H. Bing, Concerning hereditarily indecomposable continua, ibid. 1 (1951), 43-51.

[4] C. R. Borges, Are EC-spaces AE(metrizable)?, Colloq. Math. 62 (1991), 135-143.

[5] R. Cauty, Un espace métrique linéaire qui n'est pas un rétracte absolu, Fund. Math. 146 (1994), 85-99.

[6] C. O. Christenson and W. L. Voxman, Aspects of Topology, Dekker, New York, 1977.

[7] J. Dugundji, An extension of Tietze's Theorem, Pacific J. Math. 1 (1951), 353-367.

[8] —, Topology, Allyn and Bacon, Boston, 1966.

[9] K. Eda, Free $\sigma$-products and noncommutatively slender groups, J. Algebra 148 (1992), 243-263.

[10] K. Eda and K. Sakai, A factor of singular homology, Tsukuba J. Math. 15 (1991), 351-387.

[11] R. Engelking, General Topology, Heldermann, Berlin, 1989.

[12] M. I. Graev, Free Topological Groups, Izv. Akad. Nauk SSSR Ser. Mat. 12 (1948), 279-324 (in Russian); English transl.: Amer. Math. Soc. Transl. 8, 305-364.

[13] E. Hewitt and K. Ross, Abstract Harmonic Analysis I, Springer, Berlin, 1963.

[14] E. Michael, Some extension theorems for continuous functions, Pacific J. Math. 3 (1953), 789-806.

[15] M. G. Tkachenko, On completeness of free abelian topological groups, Soviet Math. Dokl. 27 (1983), 341-345.

[16] J.van der Bijl and J.van Mill, Linear spaces, absolute retracts, and the compact extension property, Proc. Amer. Math. Soc. 104 (1988), 942-952.

DEPARTMENT OF INFORMATION

WASEDA UNIVERSITY

TOKYO, 169 JAPAN

E-mail: EDA@LOGIC.INFO.WASEDA.AC.JP 